\title{
Helminth-induced alterations of the gut microbiota exacerbate bacterial colitis
}

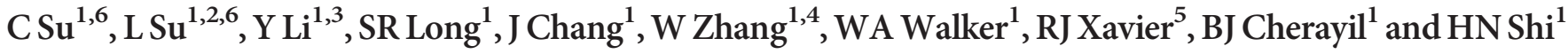

Infection with the intestinal helminth parasite Heligmosomoides polygyrus exacerbates the colitis caused by the bacterial enteropathogen Citrobacter rodentium. To clarify the underlying mechanism, we analyzed fecal microbiota composition of control and helminth-infected mice and evaluated the functional role of compositional differences by microbiota transplantation experiments. Our results showed that infection of Balb/c mice with $\mathrm{H}$. polygyrus resulted in significant changes in the composition of the gut microbiota, characterized by a marked increase in the abundance of Bacteroidetes and decreases in Firmicutes and Lactobacillales. Recipients of the gut microbiota from helminth-infected wide-type, but not STAT6-deficient, Balb/c donors had increased fecal pathogen shedding and significant worsening of Citrobacter-induced colitis compared to recipients of microbiota from control donors. Recipients of helminth-altered microbiota also displayed increased regulatory T cells and IL-10 expression. Depletion of CD4 ${ }^{+} \mathrm{CD} 25^{+} \mathrm{T}$ cells and neutralization of IL-10 in recipients of helminth-altered microbiota led to reduced stool C. rodentium numbers and attenuated colitis. These results indicate that alteration of the gut microbiota is a significant contributor to the $H$. polygyrus-induced exacerbation of $C$. rodentium colitis. The helminth-induced alteration of the microbiota is Th2dependent and acts by promoting regulatory T cells that suppress protective responses to bacterial enteropathogens.

\section{INTRODUCTION}

The gastrointestinal tract is colonized by an enormous community of microbes, the microbiota, that is composed of thousands of bacterial species and that plays an important role in health and disease. ${ }^{1}$ Recent evidence indicates a major role for the microbiota in immune system development. ${ }^{2-4}$ The microbiota also contributes to the maintenance of the gut epithelial barrier and provides resistance to colonization by enteropathogens. ${ }^{5}$ These beneficial effects depend on complex, mutualistic interactions between the gut microbiota, the intestinal epithelium, and the host immune system. Circumstances that disrupt these interactions can have significant effects on the development of a wide spectrum of disorders. ${ }^{1,6-9}$

Helminth infections form a major group of tropical diseases that affect about one third of the world's population. ${ }^{10}$ In addition to the primary pathological effects of the worms themselves, helminth infection has been implicated in secondary abnormalities such as increased susceptibility to other pathogens, ${ }^{11-13}$ impaired responses to vaccination and nutritional derangements. ${ }^{14-16}$ The fact that the geographic distribution of several helminth infections coincides with many devastating microbial diseases such as AIDS, malaria, and tuberculosis could be a reflection of helminth-induced alterations in host physiology. An effective intervention for prevention and control of helminth infections is deworming. ${ }^{17,18}$ However, deworming does not always alter the occurrence of the secondary abnormalities associated with helminth infection. For example, although deworming improved tuberculin-stimulated $\mathrm{T}$-cell proliferation and IFN- $\gamma$ production by PBMCs from helminth-infected patients, ${ }^{19}$ it did not affect the clinical outcome of tuberculosis in helminth co-infected patients. ${ }^{20}$

We have been characterizing the effects of helminth infection on susceptibility to other pathogens using a mouse model that

${ }^{1}$ Mucosal Immunology and Biology Research Center, Massachusetts General Hospital and Harvard Medical School, Charlestown, Massachusetts, USA. ${ }^{2}$ Department of Veterinary Medicine, College of Veterinary Medicine, Jilin University, Changchun, Jilin, China. ${ }^{3}$ College of Animal Science, Zhejiang University, Hongzhou, Zhejiang, China. ${ }^{4}$ Research Center for High Altitude Medicine, Qinghai University School of Medicine, Xining, Qinghai, China and ${ }^{5}$ Gastrointestinal Unit and Center for the Study of Inflammatory Bowel Disease, Massachusetts General Hospital and Harvard Medical School, Boston, Massachusetts, USA. Correspondence: HN Shi (shiha@helix.mgh.harvard.edu).

${ }^{6}$ The first two authors contributed equally to this work.

Received 2 February 2016; accepted 22 February 2017; published online 29 March 2017. doi:10.1038/mi.2017.20 
makes use of the intestinal helminth parasite Heligmosomoides polygyrus and the Gram-negative bacterial enteropathogen Citrobacter rodentium. We have shown that co-infection with $H$. polygyrus exacerbates intestinal inflammation caused by the bacterial pathogen via a signal transducer and activator of transcription 6 (STAT6)-dependent mechanism. ${ }^{11,21-23}$ This exacerbation was associated with enhanced Citrobacter output in the stool and increased translocation of the bacteria to systemic tissues. We considered the possibility that some of these effects were caused by helminth induced alterations in the gut microbiota. Although recent evidence indicates that helminth infection can lead to alterations of gut microbiota composition, ${ }^{24,25}$ the functional consequences of these alterations, including their contribution to the modulation of host susceptibility to bacterial enteropathogens and the exacerbation of Citrobacter colitis, have not been investigated.

In the studies presented here, we characterized the changes in microbiota composition associated with $H$. polygyrus infection and tested their effects on the course of C. rodentium infection. We demonstrate that $H$. polygyrus infection induces dramatic abnormalities in the composition of the intestinal microbiota that are dependent on the Th2 immune skewing caused by the helminth infection and that are not corrected by eliminating the helminth with anti-helminthic treatment. Moreover, we show by means of microbiota transplantation experiments that the helminth-altered microbiota is sufficient (in the absence of the worm itself or potential host immune factors in the intestinal contents) to dysregulate mucosal immune responses and confer increased susceptibility to Citrobacter colitis on naïve animals. Our results indicate that alterations in the gut microbiota contribute significantly to the pathological consequences of helminth infection, and suggest that helminths may exert at least some of their immunoregulatory effects on host protective immunity against bacterial enteropathogens by influencing microbiota composition.

\section{RESULTS}

\section{H. polygyrus infection significantly alters micobiome composition in Balb/c mice}

We recently showed that $H$. polygyrus infection worsened the course of a concurrent infection with $C$. rodentium, with significantly increased and prolonged bacterial output. ${ }^{11,22,26}$ To determine whether this effect might be caused by helminthinduced alterations in the gut microbiota, we used bacterial $16 \mathrm{~S}$ rRNA gene sequencing to compare the stool microbiome composition of control and helminth-infected mice in two independent experiments consisting of 3 mice per group. Our pooled data revealed that $H$. polygyrus induced significant changes in the gut microbiota (Figure 1a-e). At the genus level, a marked increase in Bacteroides and a decrease in Lactobacillus were detected in helminth-infected mice compared to the uninfected controls (Figure 1a). Marked differences were also observed at the species level (Figure 1b). Analysis of overall richness (i.e., number of distinct organisms present within the microbiome), expressed as the number of operational taxonomic units, revealed that it differed significantly between control and $H$. polygyrus-infected mice (Table 1). These results demonstrate that $H$. polygyrus infection results in significant changes in gut microbiota composition, characterized by a marked increase in the abundance of the bacterial phylum Bacteroidetes and a decrease in Firmicutes (Clostridium, Lactobacillus and Eubacterium; Figure 1d). Helminths may change microbiota composition through the effects of parasite products or through the immune responses elicited by the parasite. Our next set of experiments directly tested the impact of the worms on the gut microbiota by deworming some of the infected mice using our published methods, which can effectively clear the worms, ${ }^{27}$ and comparing the gut microbiome composition of helminthinfected mice with and without worm present in the gut. Our results from the microbiota analysis revealed a high degree of similarity in the gut microbiota of the control and dewormed animals (tested 1 week after the anti-helminthic treatment; Figure 1f). Further analysis of the abundance of some representative gut microbes was tested using quantitative PCR (qPCR) at 2 and 3 weeks after deworming. The results showed that the relative abundances of Bacteroides, segmented filamentous bacteria (SFB), and Lactobacillus at 2 and 3 weeks after deworming were similar to helminth-infected mice that were not dewormed (see Supplementary Figure 1a-c online). This finding suggests strongly that the mechanism by which helminth infection alters the gut microbiota is via its effect on the host immune system rather than via direct effects of the worm or worm components.

\section{Transplantation of helminth-altered microbiota recapitulates the effects of helminth infection on Citrobacter colitis}

To determine the functional significance of the microbiota changes induced by helminth infection, we used a fecal transplantation approach. Balb/c mice whose gut flora had been depleted by means of a previously reported antibiotic regimen ${ }^{28}$ were colonized with microbiota (mixture of cecal, colonic, and fecal contents) from either control or $H$. polygyrus-infected mice. The recipient mice received 3 microbiota transfers over the course of a week.

We first confirmed that the microbiota of the recipient mice reproduced the gut flora of their donors. One week after the last transfer, fecal pellets were collected from the recipients and the microbiota was analyzed using bacterial $16 \mathrm{~S}$ rRNA gene sequencing. The results showed a clear difference in microbiota composition between the two groups of recipient mice at the genus level, as evidenced by an increase in Bacteroides and decrease in Clostridium and Lactobacillus in mice colonized with microbiota from helminth-infected donors (Figure 2a,b). A clear distinction in the relative abundance of the most dominant species in the recipient mice was also detected between mice that were colonized with control microbiota and microbiota from helminth-infected mice (Figure 2c). A comparison of microbiota composition between helminthinfected donors and the corresponding recipients revealed high similarity in the pattern of the microbiota community 
Relative abundance of the top 10 genera in each sample.

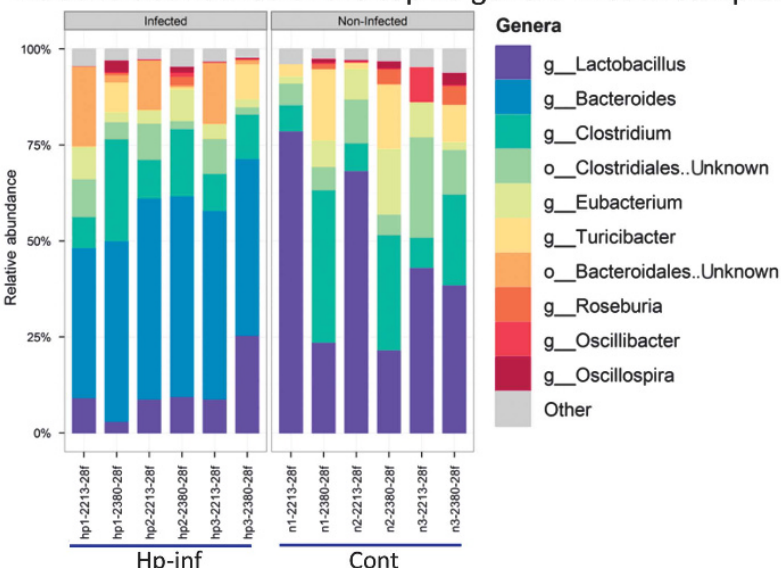

b Relative abundance of the top 15 species.

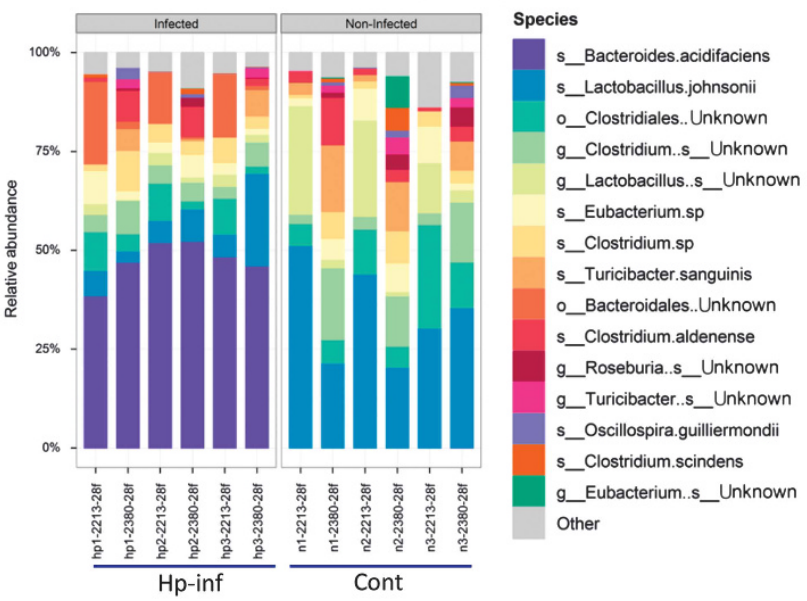

e Heatmap summarizing the relative abundance of the 10 most dominant genera

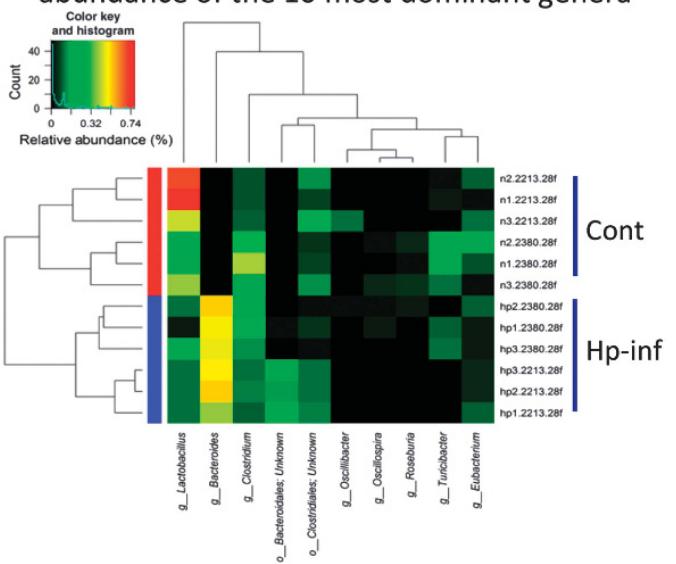

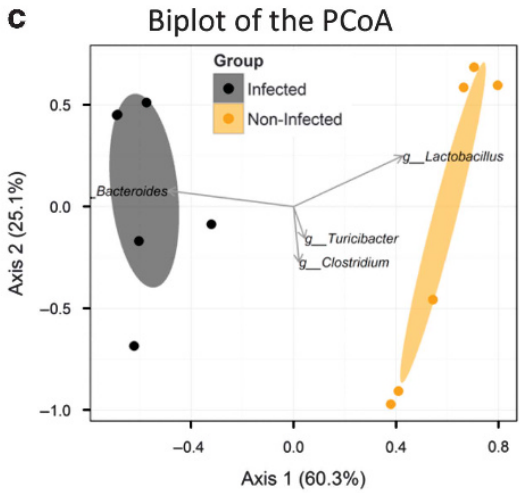

d OTUs that exhibited a significant change between infected and non-infected samples

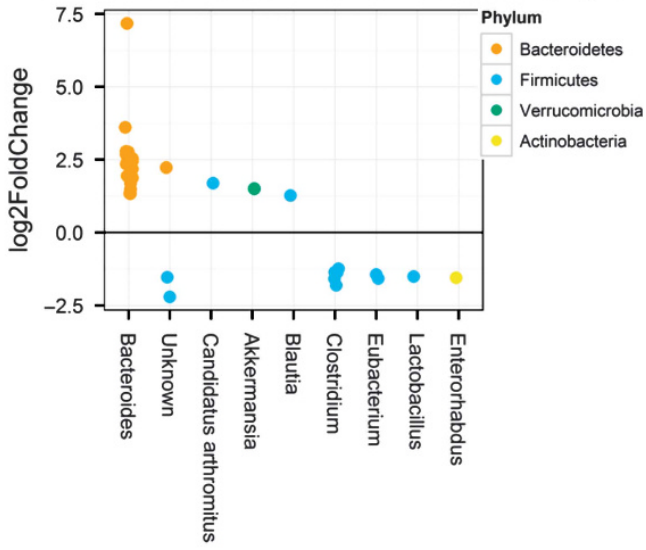

f Relative abundance of the top 10 genera in each sample indicates high similarity in the gut microbiota of helminth-infected mice with and without deworm treatment.

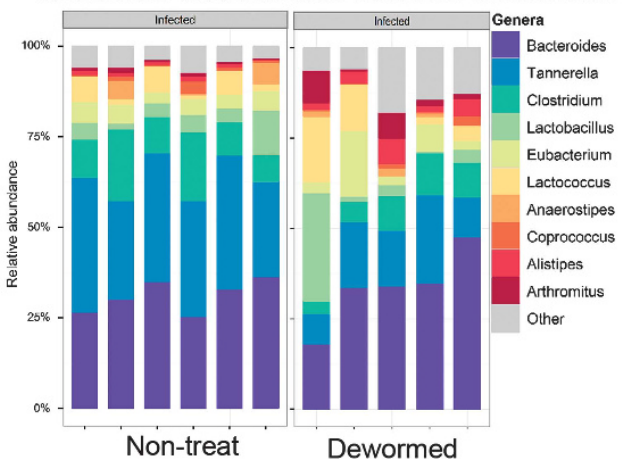

Figure $1 H$. polygyrus infection induces alterations of intestinal microbiota in Balb/c mice. The results are 16S rRNA gene sequence data from fecal pellets of control and H. polygyrus-infected Balb/c mice (2 weeks post-infection). (a) Taxonomic classifications at the genus level showing the relative abundance of the top 10 genera in each sample. (b) Relative abundance of the top 15 species in each sample. (c) Biplot of the principle coordinates analysis (PCoA), based on Bray-Curtis distances. Ellipses represent the $95 \%$ confidence interval around group centroids, and the test for group differences shows $P=0.005$. Arrows indicate the contribution of individual taxa to the PCoA axes, and only those taxa with the largest contributions are shown. (d) operational taxonomic units (OTUs) that exhibited a significant change between infected and non-infected samples. Each dot represents an individual OTU. Positive values indicate an increase relative to non-infected, and negative values indicate a decrease. OTUs are grouped by the genus $(x$-axis) in which they belong, and are color coded by phylum. (e) Heat map summarizing the relative abundance of the 10 most dominant genera. Samples are sorted based on hierarchical clustering of Bray-Curtis distances, and infection is highlighted via the colors on the left. The order of taxa is determined by a hierarchical clustering of Euclidean distances among taxa. (f) Relative abundance of the top 10 genera in each sample showed high similarity in the gut microbiota of helminth-infected mice with and without deworming treatment. 
Table 1 Analysis of variance table for bacterial species richness (number of OTUs)

\begin{tabular}{lrrrcc}
\hline & Df & Sum sq & Mean sq & $F$ value & $\operatorname{Pr}(>F)$ \\
\hline Group & 1 & 2716.65 & 2716.65 & 5.08 & 0.0481 \\
Residuals & 10 & 5363.55 & 536.35 & - & - \\
\hline
\end{tabular}

Abbreviation: OTU, operational taxonomic unit.

Overall richness (i.e., number of distinct organisms present within the microbiome) was expressed as the number of OTUs, and was quantified using the Chao1 richness estimator.
(Figure 2d). qPCR analysis confirmed the helminth infectionassociated increase in Bacteroides and SFB and decrease in Lactobacillus in the recipient mice (Figure $2 \mathbf{e}-\mathbf{g}$ ).

We then investigated the functional effects of the altered microbiota induced by $H$. polygyrus infection on the response to C. rodentium. To this end, we infected the recipients with C. rodentium 7 days after the last microbiota transfer, as illustrated in Figure 3a. We found that recipients of fecal material from helminth-infected donors had more severe

a Relative abundance of the top 10 genera in each sample of recipient mice.
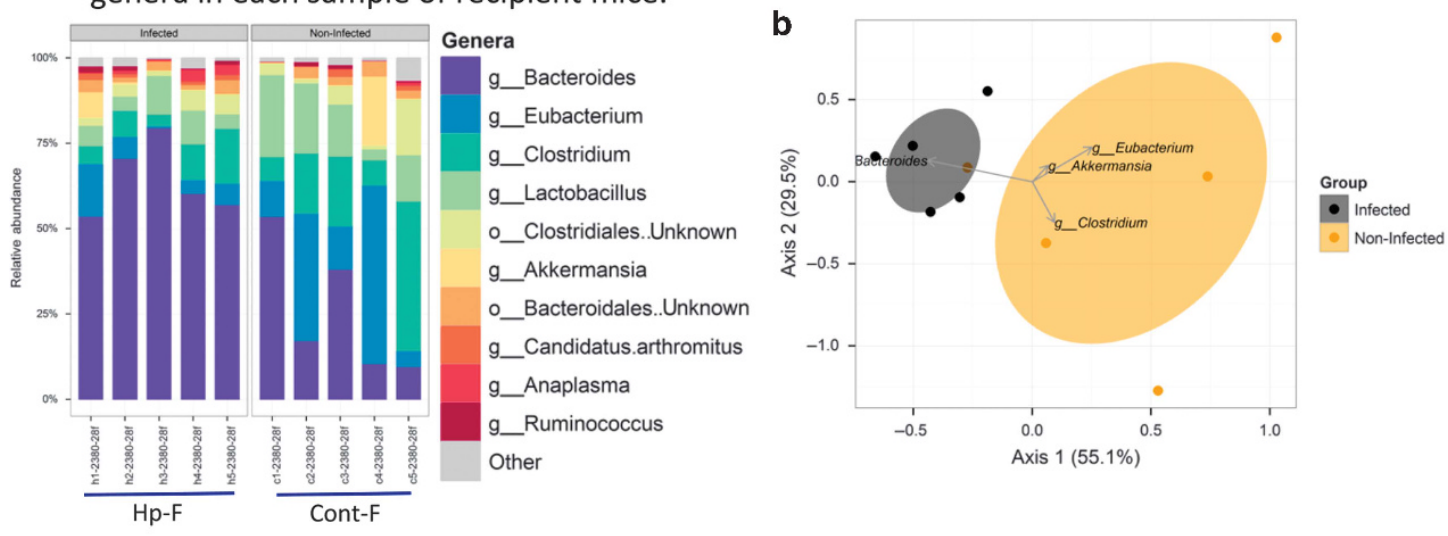
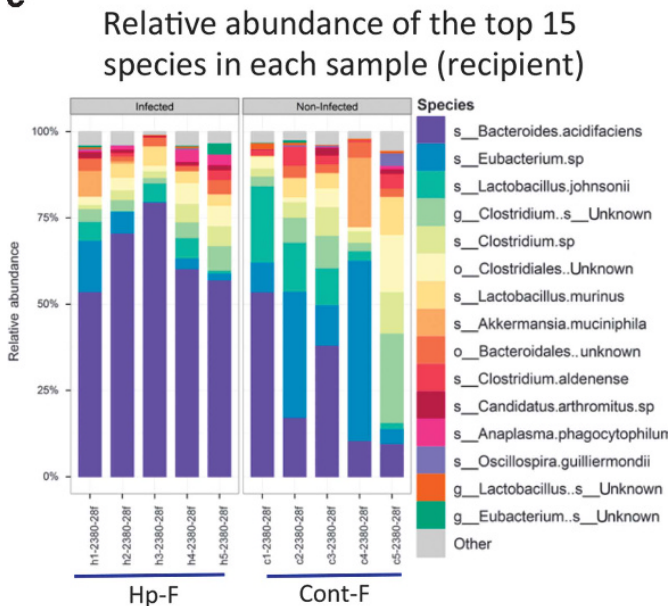

e

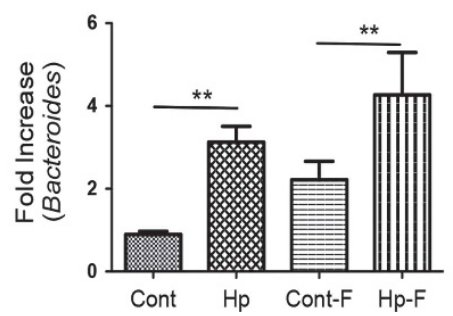

$\mathbf{f}$

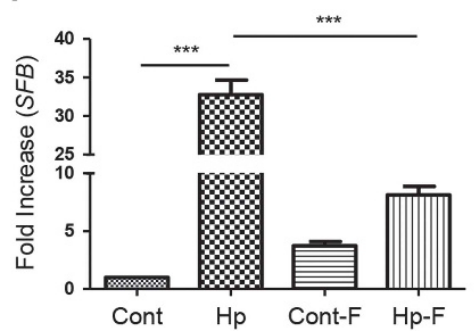

d Infected samples. Relative abundance of the top 10 genera in each sample (colonized vs.donor)
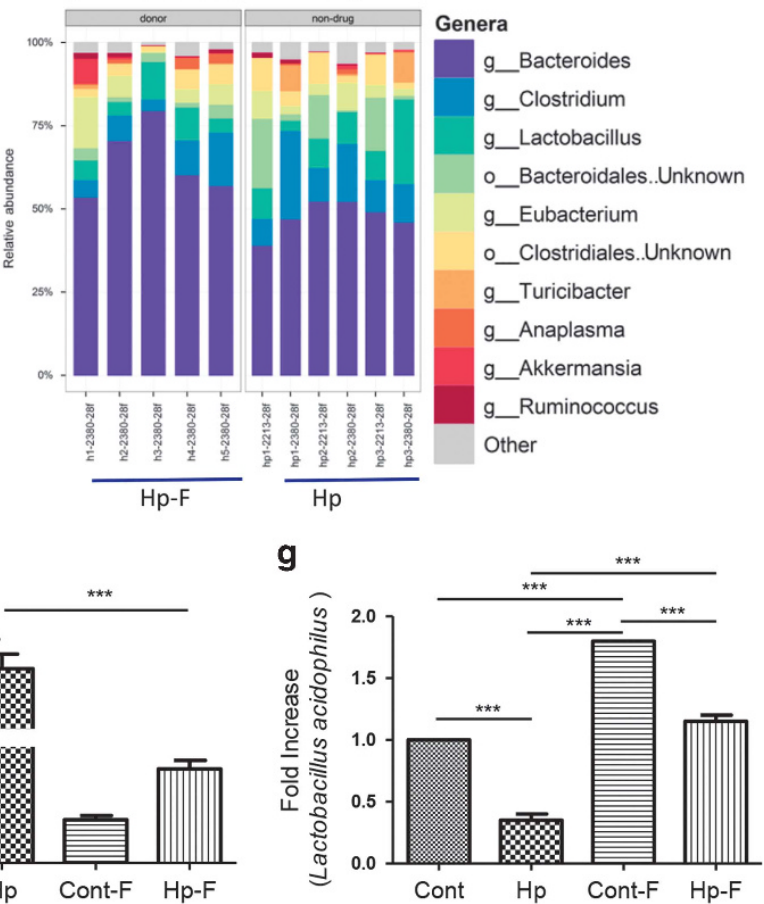

Figure 2 Colonization of recipient mice with microbiota from $\mathrm{H}$. polygyrus-infected or control mice reproduces the microbial composition of the donor. The results are 16S rRNA gene sequence data from fecal pellets of recipient mice that are colonized with microbiota from control (Cont- $F$ ) and H. polygyrus-infected (Hp-F) donor mice. (a) The relative abundance of the top 10 genera in each sample of the different recipient groups, in which mice were colonized with Hp-F or Cont-F. (b) Biplot of the principle coordinates analysis (PCoA), based on Bray-Curtis distances. (c) Relative abundance of the top 15 species in each sample from the recipient mice that were colonized with $\mathrm{Hp}-\mathrm{F}$ and Cont-F. (d) The relative abundance of the top 10 genera in each sample from the Hp-F-colonized recipient mice and from helminth-infected donor (Hp). (e- $\mathbf{g})$ qPCR analysis of fecal Bacteroides, SFB, and Lactobacillus level in donor mice: normal and H. polygyrus-infected mice, and in the recipient mice that are colonized with Cont-F and Hp-F. 


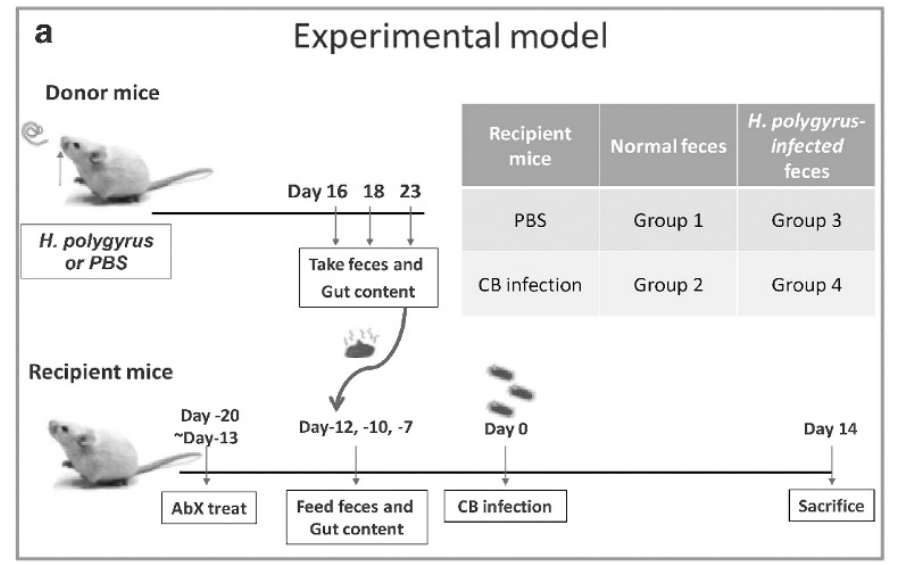

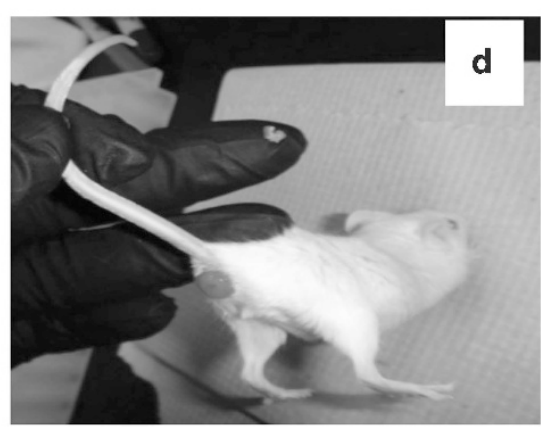

Hp-F + C.r.
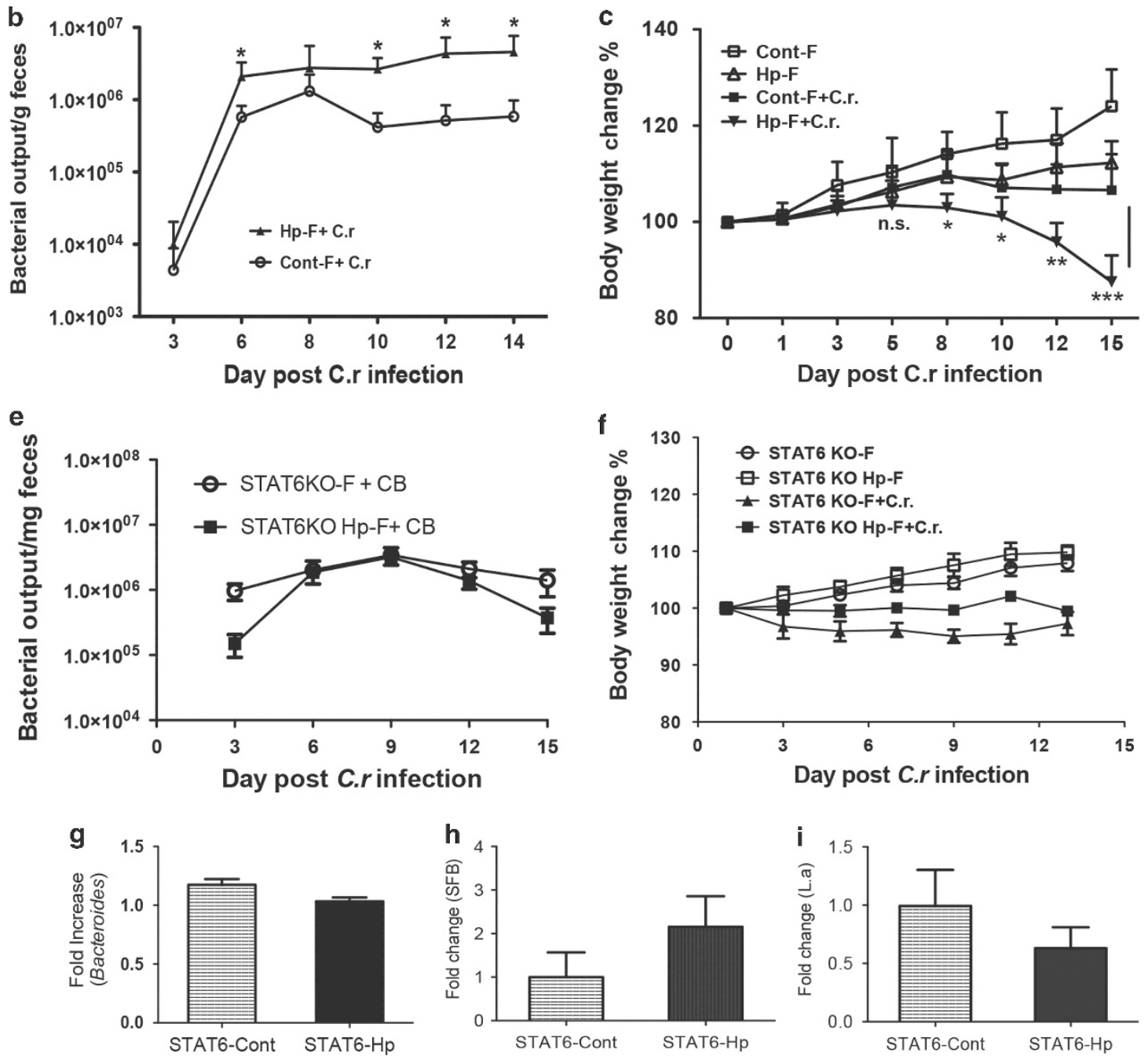

Figure 3 Colonization of mice with microbiota from H. polygyrus-infected mice results in exacerbated C. rodentium infection. (a) Microbiota colonization protocol. (b) Colonization of mice with $\mathrm{Hp}-\mathrm{F}$ results in increased fecal $\mathrm{C}$. rodentium output in mice. (c) Body weight changes of recipients of Cont- $\mathrm{F}$ and $\mathrm{Hp}-\mathrm{F}$ with and without $\mathrm{C}$. rodentium infection. (d) Development of rectal prolapse in mice colonized with $\mathrm{Hp}-\mathrm{F}$ after $\mathrm{C}$. rodentium infection. (e) Fecal C. rodentium output in mice colonized with microbiota from uninfected (control) or H. polygyrus-infected STAT6 KO mice (STAT6KO Hp-F). (f) Body weight changes of mice colonized with STAT6KO-F or STAT6KO Hp-F with and without $C$. rodentium infection. Data shown are pooled from three independent experiments and are expressed as a percentage of the initial body weight \pm s.e. $(n=10-15)$ at each time point. (g-i): qPCR analysis of fecal Bacteroides, SFB, and Lactobacillus level in STAT6 KO mice with and without $H$. polygyrus infection. STAT6, signal transducer and activator of transcription 6 . 
Citrobacter colitis when compared to recipients of fecal material from uninfected donors. The enhanced disease severity was indicated by increased fecal bacterial pathogen output (Figure 3b), greater body weight loss (Figure 3c), and an increased incidence of rectal prolapse $(\sim 10 \%$ in recipients of microbiota from helminth-infected donors-Figure 3dversus $0 \%$ in recipients of microbiota from uninfected donors).

Helminth infections are known to induce Th2-type immune responses in the host. To determine the role of the helminthinduced Th2 response in modifying the microbiota, we infected Th2-deficient STAT6 knockout (KO) mice with $H$. polygyrus, collected the microbiota from both infected and uninfected STAT6 KO donors, and transferred to the antibiotic-pretreated wild-type recipient mice, which were then infected with C. rodentium. In contrast to our findings with transfer of microbiota from helminth-infected wild-type mice (Figure 3a-c), microbiota from helminth-infected STAT6 $\mathrm{KO}$ donors failed to worsen the severity of $C$. rodentium infection as indicated by body weight changes (Figure 3f) and fecal bacterial pathogen output (Figure 3e). These results suggest that an intact Th2 response is required for the helminth-induced alterations in the microbiota that are involved in exacerbation of Citrobacter infection. This notion is supported by stool qPCR analysis, which showed that there was no significant effect of the $H$. polygyrus infection on the abundance of some representative gut microbes (Bacteriodes, SFB, and Lactobacillus) in the stool of the STAT6 KO mice (Figure 3g-i). These results not only support our statement that helminth infection alters the gut microbiota via effects on the immune system but also demonstrate that a specific aspect of immunity, i.e, an intact Th2 response, is required for the helminth-induced alterations in the microbiota that are involved in exacerbation of Citrobacter infection.

Our previously published work demonstrated that coinfection with $H$. polygyrus resulted in enhanced Citrobacter-associated colonic pathology in mice, including heightened colonic crypt elongation, massive cellular infiltration of the colonic lamina propria, epithelial erosions, and edema of the gut wall. ${ }^{11,26}$ In the current study, we followed up on these earlier observations to determine the impact of helminthaltered microbiota on Citrobacter-induced intestinal inflammation and tissue damage. Macroscopic examination of the intestine of recipient mice following $C$. rodentium infection showed that colonic thickening was more pronounced in the recipients of microbiota from helminth-infected donors than in the recipients of control microbiota (Figure 4a). Microscopic examination revealed that mice colonized with control microbiota and infected with C. rodentium showed typical pathological changes, including thickening of the wall of the colon, colonic epithelial cell hyperplasia, and crypt elongation (Figure $\mathbf{4} \mathbf{c}, \mathbf{d}$ ). Recipients of microbiota from helminth-infected donors showed more severe pathology, including massive cellular infiltration of the lamina propria and epithelial erosions (Figure 4e,f). The pathology scores for inflammation and intestinal damage were significantly higher in the recipients of microbiota from helminth-infected donors than in recipients of control microbiota (Figure $\mathbf{4 b}$ ). These results are in line with our earlier findings in $\mathrm{H}$. polygyrusC. rodentium co-infected mice. ${ }^{11,26}$ We next examined whether helminth-induced alterations in the microbiota affect the goblet cell response during $C$. rodentium infection by using periodic acid-Schiff staining to identify goblet cells. The results showed a significant increase of goblet cells in Citrobacterinfected recipients of microbiota from helminth-infected donors relative to Citrobacter-infected recipients of control microbiota (Figure $\mathbf{4 g - i}$ ). Taken together, our results indicate that transfer of microbiota from $H$. polygyrus-infected donors faithfully recapitulates almost all the previously described effects of the helminth itself on the course of Citrobacter infection. ${ }^{11,26}$

As our microbiota transfer experiments were conducted using whole intestinal contents, there is a formal possibility that the effects of the transfer were mediated by factors other than the actual commensal microbes (e.g., host immune molecules present in the intestinal lumen). We addressed this possibility using two experimental approaches. In the first, the donor fecal material was centrifuged to separate the microorganisms and other particulates from any soluble material. The pellet was washed and then transferred to the recipients. In the second approach, the supernatant of the centrifuged fecal material was filtered through a $0.2-\mu$ filter and the filtrate (free of microbes and insoluble material) was transferred to the recipients. We confirmed that the centrifuged pellet, as well as the unfractionated fecal material, contained large numbers of culturable bacteria, whereas the filtrate did not have any culturable bacteria (Figure 5a). The recipient mice were infected with $C$. rodentium and their responses were determined. Based on body weight changes, colon histology and colon IL-6 expression (Figure 5b-e), the recipients of both the unfractionated intestinal contents and the centrifuged pellet from helminth-infected donors developed more severe Citrobacter colitis relative to recipients of fecal material from control donors. In contrast, the severity of Citrobacter colitis in the recipients of the microbe-free filtrate of intestinal content from helminth-infected donors was similar to that in recipients of control fecal material. These results confirm that the intestinal commensal microorganisms are responsible for transferring the effects of helminth infection, and that soluble factors present in the intestinal contents are unlikely to be involved.

\section{Colonization of mice with microbiota from helminth-infected donors results in dysregulated anti-microbial responses during $\boldsymbol{C}$. rodentium infection} The functionality of the cells of the intestinal epithelium can be significantly affected by the gut microbiota. Mucosal antimicrobial peptides (AMPs) are important components of mucosal innate immunity and are generally expressed in the intestinal lining in close contact with the gut microflora. The expression of AMPs can be amplified by IL-17 and IL-22. ${ }^{29,30}$ IL-22 has been suggested to help prevent dissemination of 


\section{ARTICLES}
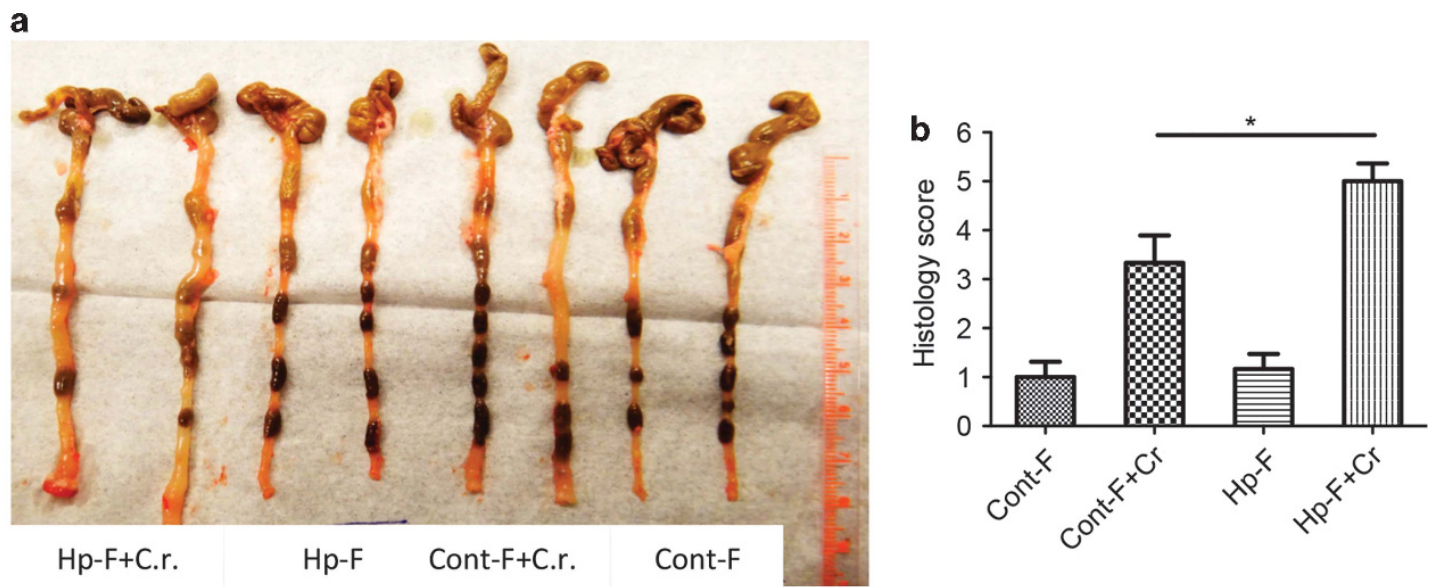

C

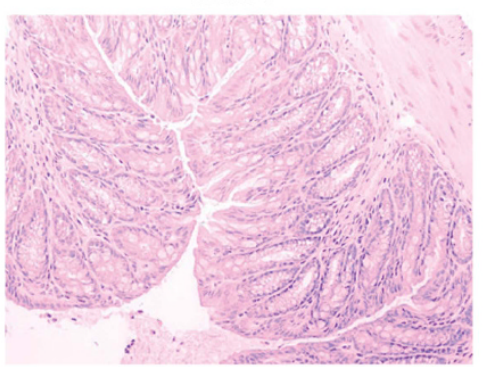

e

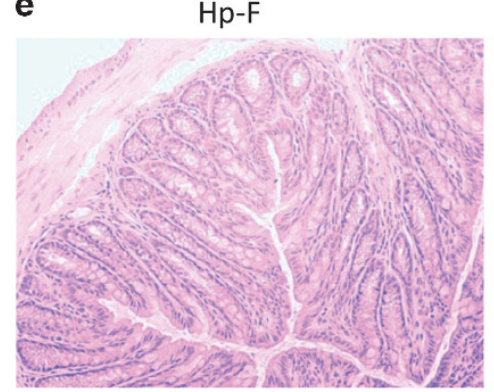

g

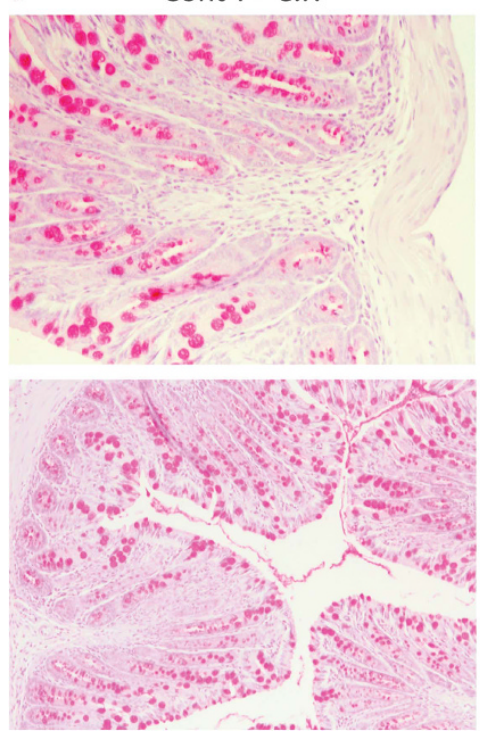

d

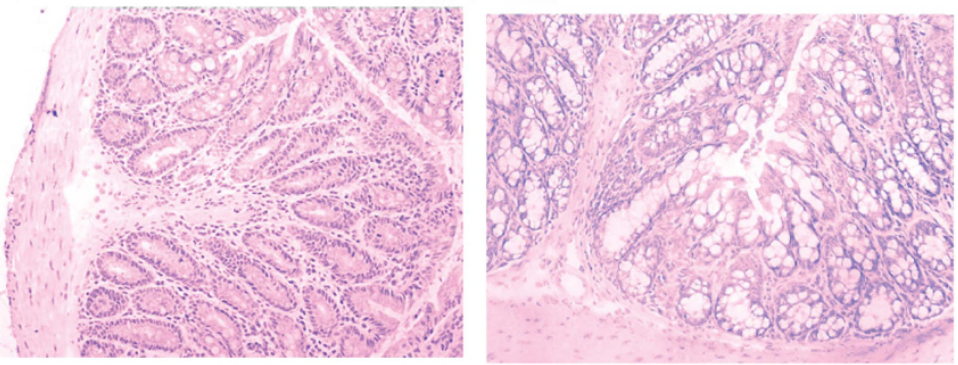

f

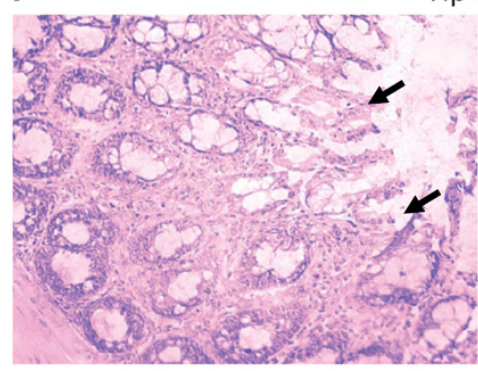

Hp-F+C.r.

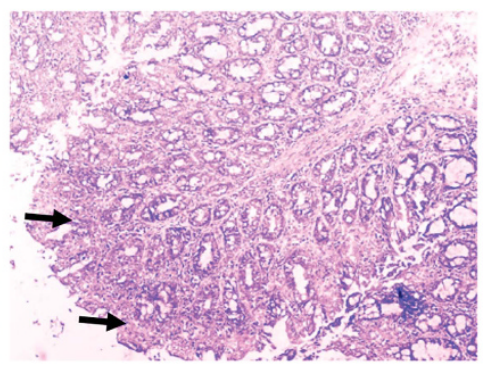

h

Hp-F+C.r.
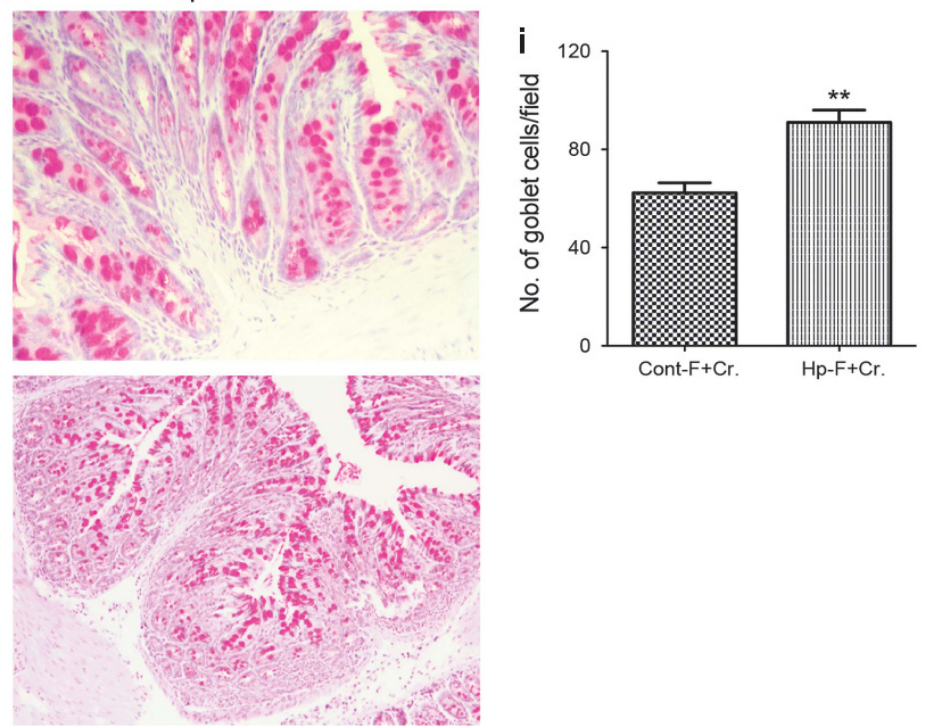
enteric pathogenic bacteria such as $C$. rodentium. ${ }^{31} \mathrm{We}$ determined if the alteration of the gut microbiota induced by $H$. polygyrus might affect mucosal innate defense by examining RNA expression of IL-22 and the AMPs Regenerating Islet-Derived 3 (Reg3) $\gamma$ and cathelicidin (CRAMP), both of which have been shown to play protective roles in C. rodentium infection. ${ }^{31,32}$ Colonic tissues from C. rodentiuminfected recipients of control microbiota displayed significant up-regulation of Reg $3 \gamma$ and CRAMP (Figure 6a,b). In contrast, C. rodentium-induced expression of Reg $3 \gamma$ and CRAMP was significantly reduced in recipients of microbiota from H. polygyrus-infected donors (Figure 6a,b). Similarly, colonic IL-22 expression was up-regulated in C. rodentiuminfected recipients of control microbiota (Figure 6c) whereas this up-regulation was reduced, albeit not to the level of statistical significance, in recipients of microbiota from helminth-infected donors (Figure $\mathbf{6 c}$ ). C. rodentium infection also typically up-regulates inducible nitric oxide synthase (iNOS) expression in the colon and loss of iNOS expression has been shown to cause a significant delay in C. rodentium a

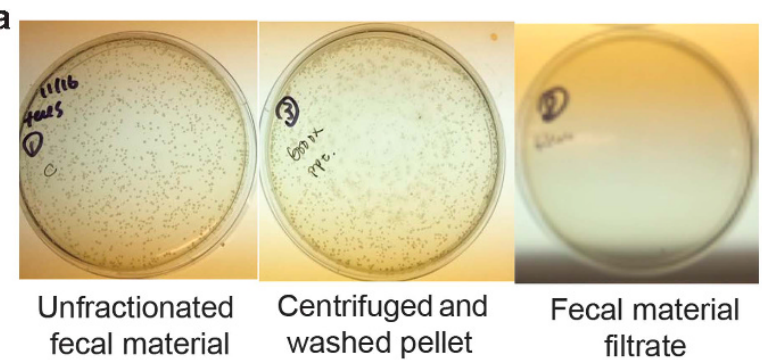

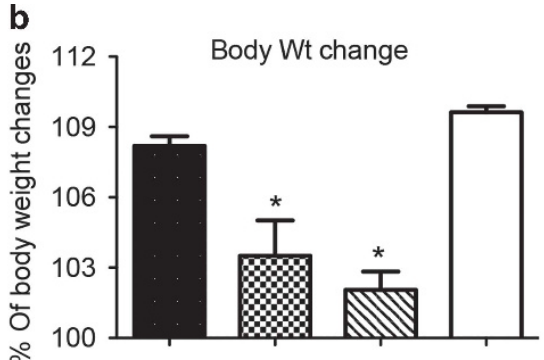

C
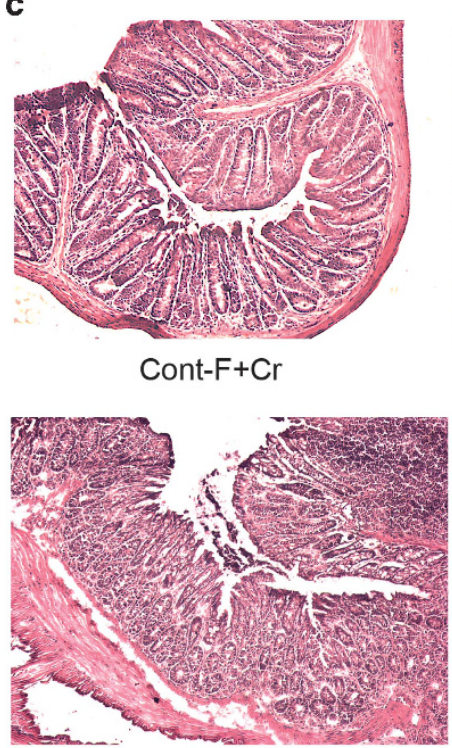

Hp-F-pellet+Cr

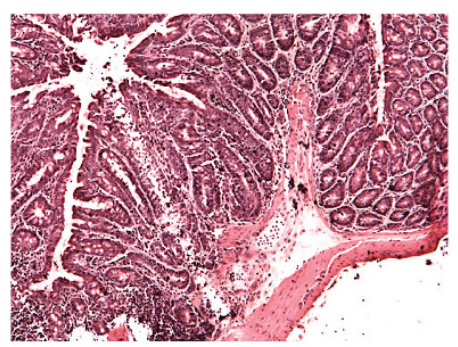

$\mathrm{Hp}-\mathrm{F}+\mathrm{Cr}$

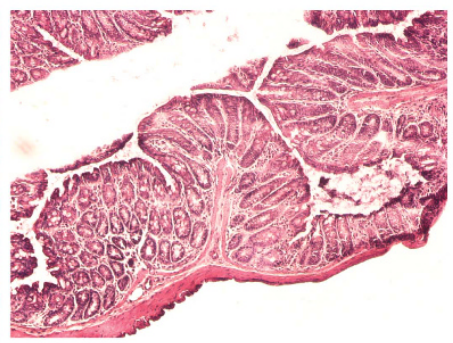

$\mathrm{Hp}-\mathrm{F}-\mathrm{Filter}+\mathrm{Cr}$
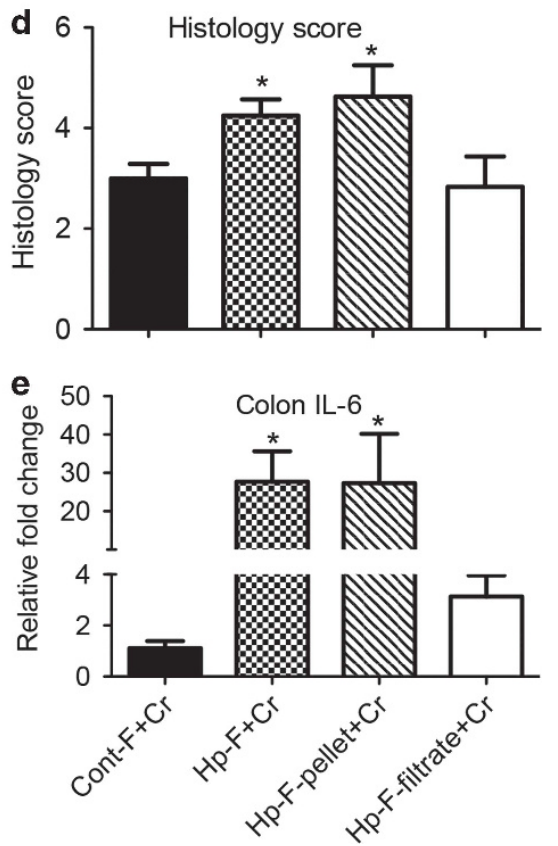

Figure 5 Transfer of intestinal microbes from helminth-infected donors enhances bacterial colitis. (a) Bacterial culture of unfractionated fecal material, and the centrifuged pellet and filtrate from the fecal material. (b-e). Body weight changes, colon pathology, and colon IL-6 expression of recipients of fecal material from normal control donor (Cont-F) or $\mathrm{H}$. polygyrus-infected donor derived unfractionated fecal material (Hp- $\mathrm{F}$ ), centrifuged pellet (Hp-F-pellet) or fecal material filtrate (Hp-F-filtrate) at 2 weeks post $C$. rodentium infection. Colonic IL-6 expression was determined by qRT-PCR. Values are the fold increase relative to $C$. rodentium infected mice colonized with normal microbiota. The data shown are the mean \pm the $\mathrm{s} . \mathrm{e} . \mathrm{m}$. $(n=3-5 \mathrm{mice} / \mathrm{group}$ ). ${ }^{\star} P<0.05$.

Figure 4 Colonization of mice with microbiota from $H$. polygyrus-infected mice exacerbates $C$. rodentium-induced colitis. (a) Colon tissues were removed from mice colonized with Cont-F or Hp-F with and without $C$. rodentium infection (2 weeks post-bacterial-infection). (b-f) Histopathological score and pathology analysis of colon. The data shown are pooled from three independent experiments with total $\left(n=9\right.$ to 12 per group). ${ }^{\star} P<0.005$. Magnification, $\times$ 200. (c-f): H\&E staining of colon section: mice colonized with Cont-F (c) or Hp-F (e) without $C$. rodentium infection. (d and f): duplicate samples are presented from C. rodentium infected mice colonized with Cont- $\mathbf{F}+\mathrm{Cr}(\mathbf{d})$ or Hp- $\mathrm{F}+\mathrm{Cr}(\mathbf{f})$. ( $\mathbf{g}-\mathbf{i})$ paraffin-embedded colon tissues from mice colonized with Cont- $\mathrm{F}(\mathbf{g})$ or Hp-F $(\mathbf{h})$ with $C$. rodentium infection (2 weeks post-bacterial-infection) were processed with periodic acid-Schiff (PAS) stain ( $\times 200$ magnification). (i): Quantitation of goblet cells in $C$. rodentium infected colons from mice colonized with Cont-F (g) or Hp-F (h). The bars indicate the mean numbers of goblet cells per high power field counted in PAS-stained colon sections. The data shown are the mean \pm the $\mathrm{s}$.e.m. $(n=3-5 \mathrm{mice}$ per group) from one of two experiments performed showing similar results. ${ }^{\star *} P<0.01$. 

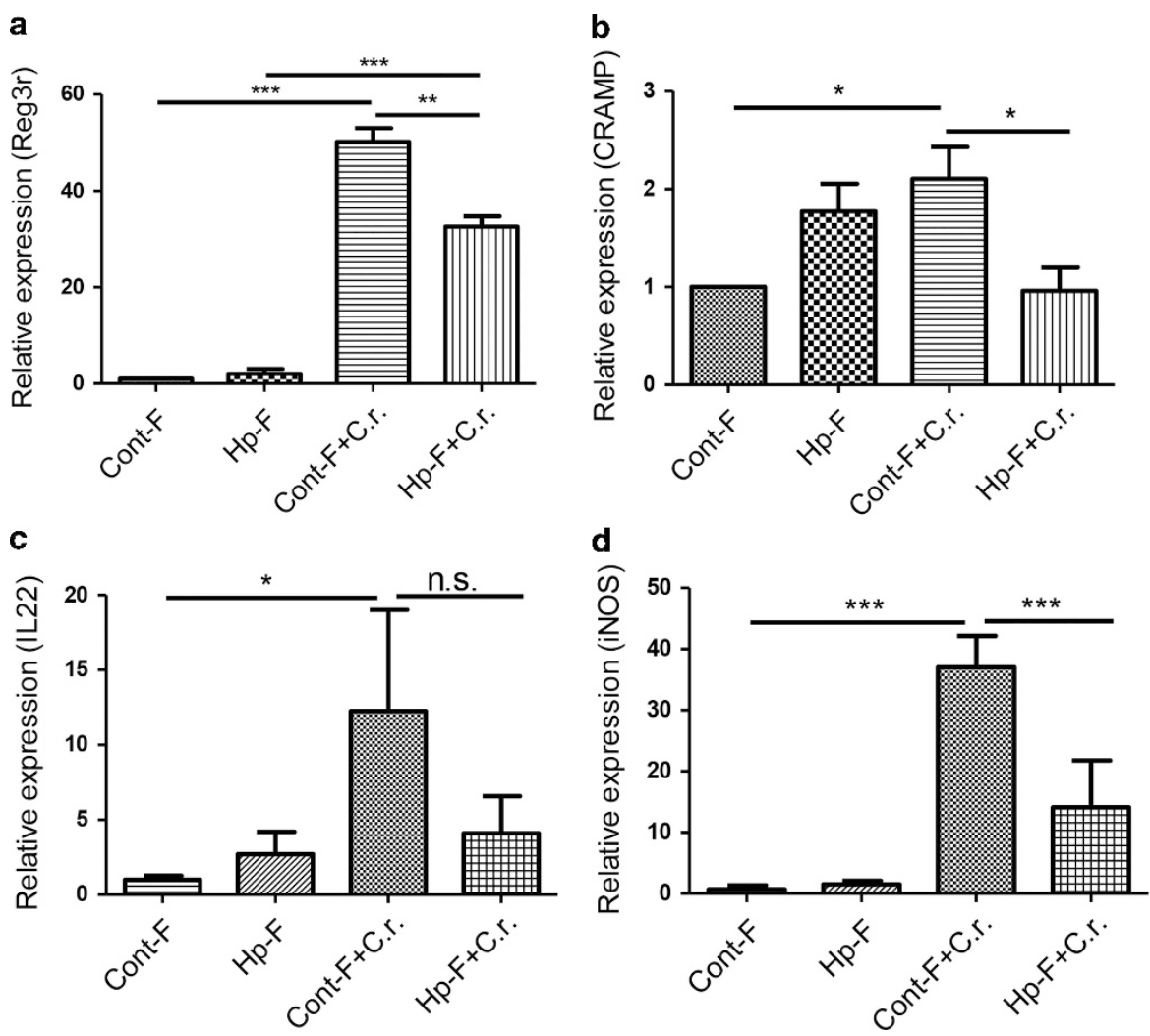

Figure 6 Colonization of mice with microbiota from helminth-infected donor mice results in down-regulation of $C$. rodentium-induced colonic antimicrobial responses. Colon tissues were collected from mice colonized with Cont- $F$ and $\mathrm{Hp}-\mathrm{F}$ with and without $C$. rodentium infection for total RNA isolation. Reg3 $\gamma(\mathbf{a})$, CRAMP (b), IL-22 (c) and iNOS (d) expression was determined using qRT-PCR. Values are the fold increase compared with baseline obtained from uninfected mice colonized with normal microbiota. The data shown are the mean \pm the s.e.m. ( $n=3-5$ mice/group) from one of three experiments performed showing similar results. ${ }^{\star} P<0.05$, ${ }^{\star \star} P<0.01,{ }^{\star \star \star} P<0.005$.

clearance. ${ }^{33}$ Quantitative reverse transcription PCR (qRTPCR) analysis of colonic tissue revealed that colonization of mice with helminth-altered microbiota resulted in suppressed bacteria-induced iNOS expression (Figure 6d).

\section{Effects of helminth-altered microbiota on Citrobacter infection are mediated by increased Tregs}

$H$. polygyrus infection induces Th2-biased immune responses, which contribute to impaired host protection against enteric bacterial infection and the induction of exacerbated bacterial colitis. ${ }^{11,26}$ We next determined whether colonization of mice with helminth-altered microbiota promotes Th2-biased immune responses to $C$. rodentium. We analyzed anti-CD3stimulated Th1, Th2, and Th17 cytokine production by mesenteric lymph node (MLN) cells using enzyme-linked immunosorbent assay. Our results showed that the production of the Th1 cytokine IFN- $\gamma$, the Th2 cytokine IL-4, and the Th17 cytokine IL-17 did not differ significantly among groups (Figure 7a), suggesting that microbiota transplantation did not influence the differentiation of these effector T cell types during C. rodentium infection.

Interestingly, our cytokine analysis revealed that both antiCD3- and Citrobacter antigen-stimulated IL-10 production by MLN cells was significantly elevated in C. rodentium-infected mice that were colonized with microbiota from helminth- infected donors (Figure 7a). Moreover, FACS analysis of MLN cells revealed an increase in the frequency of CD4 ${ }^{+} \mathrm{CD} 25^{+}$and $\mathrm{CD} 4{ }^{+}$Foxp $^{+}$cells in mice colonized with microbiota of helminth-infected donors (Figure $\mathbf{7 b}, \mathbf{c}$ ) and that these cell populations also expressed IL-10 as evidenced by the detection of higher frequencies of CD4 ${ }^{+} \mathrm{IL}_{-}-10^{+}$and, Foxp $3^{+} \mathrm{IL}-10^{+}$ cells (Figure 7c). These data suggest that microbiota from helminth-infected hosts promotes mucosal Treg responses that compromise defense against $C$. rodentium. To test this hypothesis, we repeated the microbiota transplantation experiments and treated subgroups of the recipient mice with anti-IL-10 and anti-CD25 or isotype control antibodies every other day starting 1 day before and continuing for 9 days after $C$. rodentium infection. The $\mathrm{CD} 4{ }^{+} \mathrm{CD} 25^{+}$cell population was effectively depleted as evidenced by our FACS analysis of the MLN (data not shown).

The $\mathrm{CD} 4{ }^{+} \mathrm{CD} 25^{+}$cell depletion and IL-10 neutralization did not affect fecal Citrobacter output in mice that were colonized with control microbiota (Figure 8a), but it significantly reduced fecal Citrobacter output in mice that were colonized with microbiota from helminth-infected donors (Figure 8b). Histopathology analysis of colon tissues revealed that depletion of $\mathrm{CD} 4{ }^{+} \mathrm{CD} 25^{+}$cells and IL-10 neutralization resulted in attenuated bacterial colitis (Figure 8c), which correlated with reduced colonic proinflammatory cytokine IL-6 

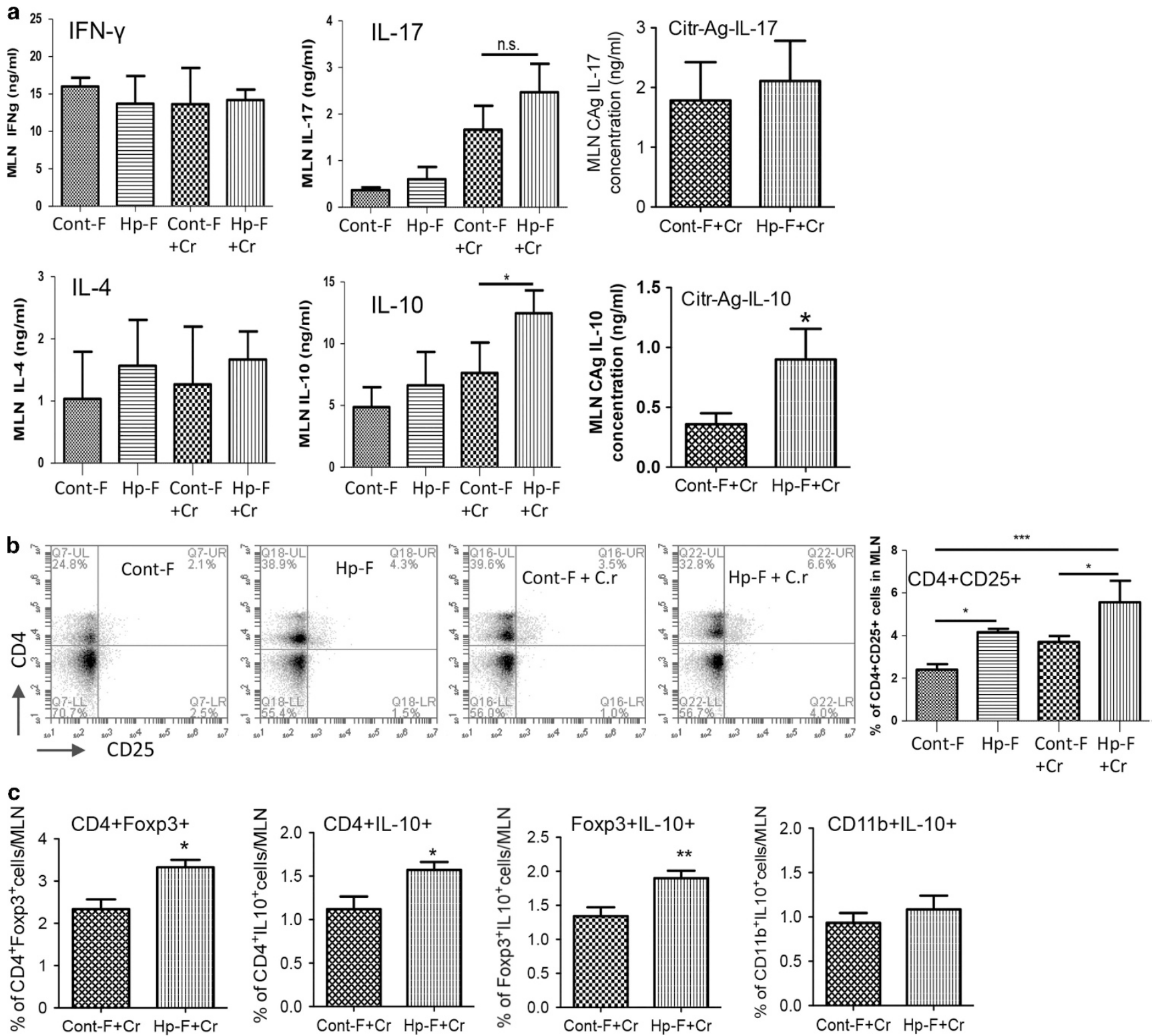

Figure 7 Effects of helminth-altered microbiota on Citrobacter infection are mediated by increased Tregs. (a) MLN cells were collected from mice colonized with Cont- $\mathrm{F}$ and $\mathrm{Hp}-\mathrm{F}$ with and without $\mathrm{C}$. rodentium infection and were cultured with surface-bound anti-CD3 mAb. Culture supernatants were collected $48 \mathrm{~h}$ later for cytokine determination (enzyme-linked immunosorbent assay). The data shows that colonization of mice with $\mathrm{Hp}-\mathrm{F}$ did not alter IFN- $\gamma$, IL-4 or IL-17 response, and it drives IL-10 response during $C$. rodentium infection. (b) FACS data shows that colonization of mice with $\mathrm{Hp}-\mathrm{F}$ induces a significant expansion of $\mathrm{CD} 4{ }^{+} \mathrm{CD} 25^{+} \mathrm{T}$ cells in MLN during $\mathrm{C}$. rodentium infection. (c) FACS data shows that colonization of mice with $\mathrm{Hp}-\mathrm{F}$ induces a significant expansion of $\mathrm{CD} 4{ }^{+} \mathrm{Foxp}^{+}, \mathrm{CD} 4^{+} \mathrm{IL}-10^{+}$, and Foxp3 ${ }^{+} \mathrm{IL}-10^{+} \mathrm{T}$ cells in $\mathrm{MLN}$ during $C$. rodentium infection. The data shown are the mean \pm the s.e.m. ( $n=3-5$ mice per group) from one of three experiments performed showing similar results. ${ }^{\star} P<0.05,{ }^{* *} P<0.01$, ${ }^{\star * *} P<0.005$.

expression (Figure 8d). These results suggest that the changes in gut microbiota induced by helminth infection act to promote Treg cell responses, thereby suppressing protection against Citrobacter and leading to enhanced bacterial infection and exacerbation of bacterial colitis.

\section{DISCUSSION}

Our previously published work demonstrated that co-infection with $H$. polygyrus worsened the course of Citrobacter infection and the associated colonic pathology in mice. ${ }^{11,26}$ In the current study, we followed up on these earlier observations to determine the impact of helminth-altered microbiota on the host response to Citrobacter. The results reported here show that $H$. polygyrus infection not only induces alterations in the intestinal microbiota composition but also that the altered gut microbiota contributes significantly to the helminth-induced exacerbation of Citrobacter infection and colitis. Although we did not directly compare recipients of microbiota from $H$. polygyrus-infected donors with $H$. polygyrus-infected mice with respect to the course of Citrobacter infection, the effects on 

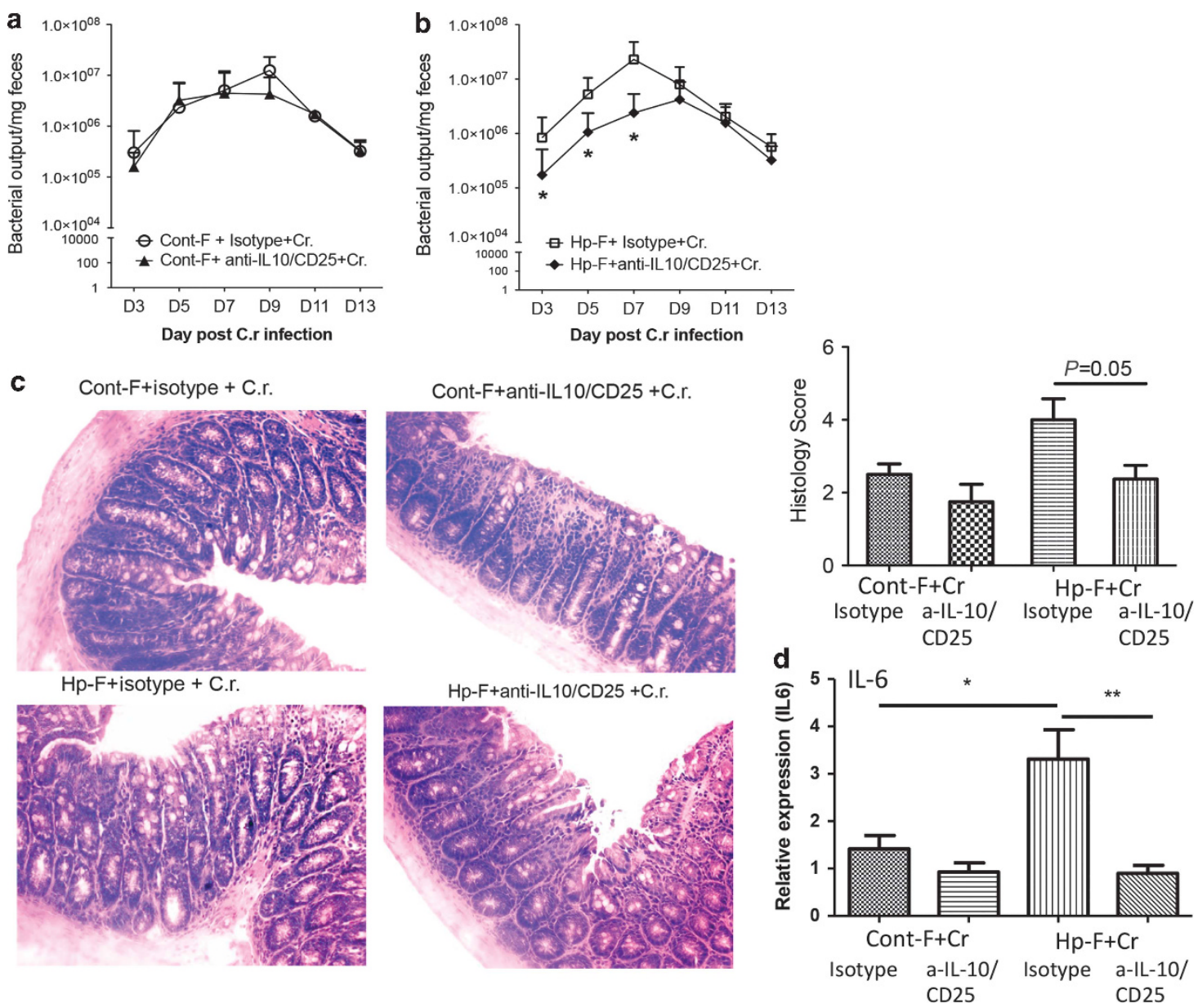

Figure 8 Treg depletion results in reduced fecal bacterial output and attenuated $C$. rodentium-mediated intestinal injury in mice colonized with microbiota from helminth-infected donor. (a,b) The numbers of Citrobacter recovered from fecal samples were determined at different time points during infection in C. rodentium-infected mice that were colonized with Cont-F or Hp-F and treated with isotype control Ab or Anti-CD25 and IL-10 Ab. (c) Histopathological analysis and score of colonic inflammation from mice infected with Citrobacter after treatment with isotype control Ab or Anti-CD25 and IL-10 Ab. (d) Colonic IL-6 expression was determined by qRT-PCR. The data shown are the mean \pm the s.e.m. ( $n=3-5$ mice/group) from one of two experiments performed showing similar results. ${ }^{*} P<0.05$.

fecal Citrobacter output and severity of colitis in the former group are very similar to what we have observed previously in $H$. polygyrus-C. rodentium co-infected animals. ${ }^{11,26}$ These results strongly suggest that altered microbiota composition is an important factor in the effects of $H$. polygyrus on Citrobacter infection and colitis. Further investigation will be required to determine the role of microbiota-independent factors in the effects of $H$. polygyrus infection. Meanwhile, our findings are consistent with other studies showing that differences in microbiota composition increase susceptibility to infections with enteropathogens such as Salmonella enterica and C. rodentium r $^{34,35}$ and also to inflammatory bowel disease (IBD). ${ }^{36,37}$ Our results also demonstrate that the mechanism by which the altered microbiota influences Citrobacter infection involves an increase in Tregs that appears to compromise anti-microbial defenses in the gut.

Helminths are well known to induce Th2 responses, and we previously demonstrated that co-infection with $H$. polygyrus exacerbates Citrobacter colitis through a STAT6 (Th2)dependent mechanism. ${ }^{11,22}$ Based on our analysis of IL-4 production by polyclonally stimulated MLN T cells, colonization with microbiota from helminth-infected donors did not appear to induce a Th2-skewed response in the recipient mice. However, there was a clear increase in colon goblet cell number in Citrobacter-infected recipients of fecal material from helminth-infected donors. Intestinal goblet cell hyperplasia is typically seen as part of a Th2 response, and it is currently not clear how transfer of the helminth-altered microbiota influences the differentiation and/or survival of goblet cells. Clarifying this issue could be an interesting area to investigate in the future. There were also no differences in Th1 and Th17 responses between recipients of control and helminth-altered microbiota during C. rodentium infection. However, colonization with the helminth-altered microbiota promoted Treg development. It was recently shown that Treg cells can promote a functional Th17 response to C. rodentium infection. ${ }^{38}$ We did not see such an effect in our study. Rather, our results suggest that helminth-induced alterations in microbiota composition disturb the balance between Treg and Th1/Th17 cells, and that the enhanced Treg response 
compromises mucosal protective responses against bacterial pathogens. This notion is supported by the results of Treg depletion, which enhanced resistance to Citrobacter infection in mice that were transplanted with helminth-altered microbiota. The enhanced resistance correlated with increased IFN$\gamma$, and to a less extent, IL-17 production in the MLN (data not shown).

The identity of the specific members of the helminth-altered microbiota or microbial metabolites that are responsible for Treg induction in the recipient mice awaits clarification. Several earlier studies have demonstrated the effects of the microbiota on the differentiation and/or maintenance of Tregs in the mouse intestine and have implicated specific microorganisms and their products in these processes. ${ }^{39}$ Interestingly, recent evidence indicates that administration of Lactobacillus taiwanensis is sufficient to elevate Treg frequencies and to render mice more susceptible to $H$. polygyrus infection. ${ }^{40}$ Recent published work also showed that helminth infection increases short chain fatty acids (SCFA) production, ${ }^{41}$ thus promoting Treg development. Thus, it seems possible that helminths may induce a gut microbiota composition that favors their own survival by promoting Treg development. A recent study also showed that transfer of the microbiota from helminth-infected mice was beneficial in an airway allergic asthma model. ${ }^{41}$ In this model, no pathogen is involved and so the immunomodulatory effects of the helminth-altered microbiota help to relieve airway inflammation. In contrast, our results indicate that the effects of the helminth-altered microbiota are detrimental, presumably because they interfere with immune responses that are required for Citrobacter clearance. Our results, therefore, indicate that a by-product of this altered microbiota composition is impaired host protective immunity against bacterial enteropathogens.

There are some similarities and discrepancies between our findings and published results on $H$. polygyrus-induced microbiota alterations. Our results are consistent with another study that showed increased abundance of the Bacteroides/ Prevotella group in $H$. polygyrus-infected C57BL/6 mice. ${ }^{42}$ However, our results showing a decrease in Lactobacillaceae in $\mathrm{Balb} / \mathrm{c}$ mice contrast with a study indicating that $H$. polygyrus infection increased the abundance of Lactobacillaceae in C57BL/6 mice. ${ }^{25}$ This discrepancy may be related to differences in model system, host genetic background, particularly since C57BL/6 mice are more susceptible to $H$. polygyrus, ${ }^{43}$ or the type of sample analyzed.

How exactly worm infection influences microbiota composition remains to be investigated. Helminths may change microbiota composition through the immune responses they elicit or through the effects of the parasites themselves. Helminth infection is known to induce Th2 and Treg responses. Our study provides strong evidence to suggest that an intact Th2 response is required for the helminth-induced alterations in the microbiota that are involved in exacerbation of Citrobacter infection, a conclusion that is supported by stool qPCR analysis showing that there was no significant effect of the $H$. polygyrus infection on the abundance of Bacteroides,
SFB, and Lactobacillus in the stool of the STAT6 KO mice (Figure $3 \mathbf{g}-\mathbf{i}$ ). In contrast, a recent study showed that IL- $4 \mathrm{R} \alpha$ $\mathrm{KO}$ mice had similar changes in microbiota composition after helminth infection, suggesting that the altered microbiota composition was independent of the IL-4/13-STAT6 signaling axis. ${ }^{42}$ Due to the profound immune regulatory capacity of helminth parasites, it is possible that other immune responses induced by helminths, such as Tregs, ${ }^{44}$ may be a potential contributor to altered gut microbiota composition. Although it still is unclear whether helminth parasites or their components may influence the gut microbiota directly, we have found in preliminary experiments that mixing a homogenate of $H$. polygyrus with fecal material from control donors failed to confer increased susceptibility to Citrobacter colitis in recipient mice (data not shown). The anti-helminthic treatment that we used in this study, which generally results in complete worm expulsion, did not correct the abnormalities of microbiota composition induced by $H$. polygyrus infection. Taken together, our results suggest that it is the altered microbiota induced by helminth infection rather than worm components that is responsible for the dysregulated mucosal immune responses that compromise protection against Citrobacter. However, the potential direct effect of the helminth parasite on the gut microbiota of the host needs to be further elucidated. Regardless, our results, together with the previously published observations mentioned above, highlight the complexity of the interactions between helminths, the microbiota and the host immune system, and the need for a better understanding of the mechanisms underlying these interactions. Our 16S rRNA gene sequencing experiments indicate that $H$. polygyrus infection results in dysbiosis of gut microbiota with marked increase in Bacteroides, which constitute the numerically predominant taxa in the murine colon, ${ }^{45}$ and decrease in Lactobacillus (Figures 2 and 3). Our qPCR experiments confirmed these observations and further indicate the change in Bacteroides, SFB abundance (Figure 2). However, the potential functional significance of these changed microbiota communities induced by helminth parasites in modulating host defense against bacterial pathogens remains to be established.

The microbiota transplantation protocol that was used in this study involves the transfer of fecal material as well as intestinal contents, which is similar to the methodology utilized in many earlier studies. ${ }^{46-48}$ However, the transferred material may contain other components, including cytokines, metabolites, etc. To exclude the possibility that some of these components may have contributed to the functional effects of the transplantation, we performed additional experiments in which the fecal material were fractionated by centrifugation or filtration to separate microorganisms from soluble components. These approaches indicated that it was the microbe-containing fraction, and not the soluble material, that transferred the effects of helminth infection on Citrobacterinduced colitis. Furthermore, we have shown clearly that the transferred material alters microbiota composition in the recipients, an outcome that is almost certainly the result of 
colonization by the donor microbiota. Based on these results, and on our finding that mixing a homogenate of $H$. polygyrus with fecal material from control donors failed to confer increased susceptibility to Citrobacter colitis in recipient mice, we feel confident that the effects we observe are caused by the microbiota and not by some other endogenous substance or worm component that might have been transferred along with the microbiota.

Taken together, the results of this study demonstrate that $H$. polygyrus infection induces significant disruption of the normal gut microbiota and that alterations in the gut microbiota are one significant contributor to helminth-induced exacerbation of bacterial colitis. The helminth-induced alteration of the microbiota acts by promoting regulatory $\mathrm{T}$ cells. Further experiments to elucidate the mechanisms involved in the helminth-induced alterations of the microbiota and the microbiota-dependent changes in Tregs and epithelial barrier functions are clearly warranted. The results of such studies will provide additional insights into the physiological consequences of parasitic infection.

\section{METHODS}

Mice and infections. Six- to 8-week-old female Balb/c mice (The Jackson Laboratory, Bar Harbor, ME) were fed autoclaved food and water, and maintained in a specific-pathogen-free facility at Massachusetts General Hospital. Animal care was provided in accordance with protocols approved by the Institutional Animal Care and Use Committee of Massachusetts General Hospital. For H. polygyrus and C. rodentium infections, we used our published protocols. ${ }^{11}$ For anthelmintic treatment, $H$. polygyrus-infected mice were treated orally with pyrantel pamoate at a dose of $172 \mathrm{mg} \mathrm{kg}^{-1}$ body weight at day 9 and 14 post infection, which effectively eliminates the parasites from the intestine. ${ }^{27}$ All animal studies were carried out in accordance with the recommendations in the Guide for the Care and Use of Laboratory Animals of the National Institutes of Health. The protocol was approved by the Sub-committee on Research Animal Care of Massachusetts General Hospital (Animal Welfare Assurance Number A3596-01).

Antibiotic treatment and microbiota transfer protocol. Balb/c recipient mice were pretreated with a cocktail of antibiotics (Kanamycin $0.4 \mathrm{mg} \mathrm{ml}^{-1}$, Gentamicin $0.035 \mathrm{mg} \mathrm{ml}^{-1}$, Colistin $850 \mathrm{U} \mathrm{ml}^{-1}$, Metronidazole $0.215 \mathrm{mg} \mathrm{ml}^{-1}$, Vancomycin $0.045 \mathrm{mg} \mathrm{ml}^{-1}$; Sigma Aldrich, St Louis, MO) in drinking water for 1 week. ${ }^{28}$ One week after antibiotic treatment, recipient mice were gavaged 3 times a week with a fecal slurry of stool pellets and cecal contents from the control (control microbiota) or $H$. polygyrusinfected (Hp-microbiota) donor mice. One week later, some of the recipient mice from both $\mathrm{Hp}$-microbiota and control microbiota colonized groups were infected with $C$. rodentium, and others left uninfected as controls. The body weight and the survival of the infected mice were measured. Fecal material was collected 3 times a week. The mice were killed 2 weeks post $C$. rodentium infection.

Intestinal content separation and transfer: 2 weeks after helminth infection, fecal material (stool pellets and cecal and colonic contents) was mixed with phosphate-buffered saline (PBS) $\left(200 \mathrm{mg} \mathrm{ml}^{-1}\right)$ and allowed to settle for $5 \mathrm{~min}$ before transfer to recipients. To remove soluble factors from the transferred material, the PBS-fecal mixture was centrifuged (at 6,000 r.p.m., $5 \mathrm{~min}$ ). The centrifuged pellets were washed with PBS and then transferred to recipients. To eliminate the microbes from the transferred material, the supernatant collected from the centrifugation of the PBS-fecal mixture was filtered through a $0.2-\mu$ filter and the filtrate was administered to recipients.

Lymphocyte isolation, measurement of cytokine production and FACS analysis. At necropsy, lymphocyte suspensions were prepared from MLN. Cells $\left(5 \times 10^{6}\right.$ cells per $\left.\mathrm{ml}\right)$ were cultured in 48 -well plates in the presence or absence of plate-bound anti-CD3 monoclonal antibody (MAb; $10 \mu \mathrm{g} \mathrm{ml}^{-1}$ ), and culture supernatants were assayed for cytokine production as previously described. ${ }^{11}$ FACS analysis was performed using our published methods. ${ }^{12}$

Quantitative detection of chemokine expression in colonic tissues. Total RNA was prepared from colon tissue using TRIzol reagent (Invitrogen Life Technologies, Carlsbad, CA) following the manufacturer's recommendations. The expression of RegIII $\gamma$, CRAMP, IL-22 and iNOS was tested by real-time quantitative RT-PCR using previously published methods. ${ }^{12}$

Histopathological examinations. At necropsy, colonic tissues were collected, frozen in Tissue Tek OCT compound (Miles, Inc., Elkhart, IN) and stored at $-80^{\circ} \mathrm{C}$. Then, $5 \mu \mathrm{m}$ sections were cut on a Leica CM1850 Cryostat (Leica Biosystem, Buffalo Grove, IL) and were stained with hematoxylin and eosin. Intestinal pathology was scored using a modified histology scoring system based on previously published methods. ${ }^{12,21}$ For goblet cell enumeration, periodic acidSchiff-stained colonic sections were prepared and digital images were collected from at least two sections in 5 mice for each group. The number of periodic acid-Schiff-positive cells per field was determined.

CD25 $^{+}$Treg depletion. To determine the impact of increased Treg responses induced by microbiota from helminth-infected host on mucosal defense against Citrobacter, the microbiota recipient mice were injected intraperitoneally (i.p.) with anti-IL-10 (BioXCell, West Lebanon, NH) and anti-CD25 (BioXCell) or isotype control antibodies (BioXCell) every other day starting 1 day before and continuing for 9 days after $C$. rodentium infection. $\mathrm{CD} 4{ }^{+} \mathrm{CD} 25^{+}$cell depletion was confirmed by FACS.

Gut microbiome analysis. Fecal pellets were collected from colons of uninfected and $H$. polygyrus-infected (2 weeks post-infection) mice at necropsy. DNA was extracted using the QIAamp DNA stool kit (QIAGEN, Chatsworth, CA). Sequencing and analysis of the stool DNA were performed at the Research and Testing Laboratory (Lubbock, TX) using 454 methods as described previously. ${ }^{49,50}$

Statistics. All in vivo experimental results were expressed as the mean \pm s.e.m. $N$ refers to the number of mice used. Statistical differences were determined using one-way analysis of variance test (Tukey's multiple comparison test) with GraphPad Prism. A $P$ value $<0.05$ was considered significant.

SUPPLEMENTARY MATERIAL is linked to the online version of the paper at http://www.nature.com/mi

\section{ACKNOWLEDGMENTS}

This work was supported by grants from the National Institutes of HealthR01 DK082427 (to H.N.S.) and by the Nutrition Obesity Research Center at Harvard (P30 DK040561). B.J.C. was supported by R01 Al089700. Libo Su, Yali Li, Shao Rong Long and Wei Zhang were sponsored by the China Scholarship Council.

\section{AUTHOR CONTRIBUTIONS}

C.S., L.S., Y.L., S.R.L., J.C. and W.Z. performed experiments; C.S. and H.N.S. designed experiments, analyzed data and wrote the paper; W.A.W., R.J.X and B.J.C. participated in editing and provided conceptual advice.

\section{DISCLOSURE}

The authors declared no conflict of interest. 


\section{REFERENCES}

1. Bäckhed, F., Ley, R., Sonnenburg, J., Peterson, D. \& Gordon, J. Host-bacterial mutualism in the human intestine. Science 307, 1915-1920 (2005).

2. Round, J. \& Mazmanian, S. Inducible Foxp3 ${ }^{+}$regulatory T-cell development by a commensal bacterium of the intestinal microbiota. Proc. Nat Acad. Sci. USA 107, 12204-12209 (2010).

3. Ivanov, I. et al. Specific microbiota direct the differentiation of IL-17producing T-helper cells in the mucosa of the small intestine. Cell Host Microbe 4, 337-349 (2008).

4. Atarashi, K. et al. Induction of colonic regulatory T cells by indigenous Clostridium species. Science 331, 337-341 (2011).

5. O'Hara, A. \& Shanahan, F. The gut flora as a forgotten organ. EMBO Rep. 7, 688-693 (2006).

6. Honda, K. \& Littman, D. The microbiome in infectious disease and inflammation. Annu. Rev. Immunol. 30, 759-795 (2012).

7. Wu, H.-J. et al. Gut-residing segmented filamentous bacteria drive autoimmune arthritis via T helper 17 cells. Immunity 32, 815-827 (2010).

8. Wen, L. et al. Innate immunity and intestinal microbiota in the development of type 1 diabetes. Nature 455, 1109-1113 (2008).

9. Maloy, K. \& Powrie, F. Intestinal homeostasis and its breakdown in inflammatory bowel disease. Nature 474, 298-306 (2011).

10. Hotez, P., Mistry, N., Rubinstein, J. \& Sachs, J. Integrating neglected tropical diseases into AIDS, tuberculosis, and malaria control. N. Engl. J. Med. 364, 2086-2089 (2011).

11. Chen, C.-C., Louie, S., McCormick, B., Walker, W. \& Shi, H. Concurrent infection with an intestinal helminth parasite impairs host resistance to enteric Citrobacter rodentium and enhances Citrobacter-induced colitis in mice. Infect. Immun. 73, 5468-5481 (2005).

12. Su, L. et al. Coinfection with an intestinal helminth impairs host innate immunity against Salmonella enterica serovar Typhimurium and exacerbates intestinal inflammation in mice. Infect. Immun. 82, 3855-3866 (2014).

13. Legesse, M., Erko, B. \& Balcha, F. Increased parasitaemia and delayed parasite clearance in Schistosoma mansoni and Plasmodium berghei co-infected mice. Acta Trop. 91, 161-166 (2004).

14. Philip, J. et al. Human infection with Ascaris /umbricoides is associated with suppression of the interleukin-2 response to recombinant cholera toxin B subunit following vaccination with the live oral cholera vaccine CVD 103HgR. Infect. Immun. 69, 1574-1580 (2001).

15. Sabin, E., Araujo, M.I., Carvalho, E.M. \& Pearce, E.J. Impairment of tetanus toxoid-specific Thl-like immune responses in humans infected with Schistosoma mansoni. J. Infect. Dis. 173, 269-272 (1996).

16. Stephenso, L. Helminth parasites, a major factor in malnutrition. World Health Forum 15, 169-172 (1994).

17. World Health Organization. Soil-transmitted helminthiasis. Weekly Epidemiological Record, World Health Organization, 85 pp. 141-148 (2010).

18. Bentwich, G. \& Bentwich, Z. Eradication of helminthic infections may be essential for successful vaccination against HIV and tuberculosis. Bull. World Health Organ. 78, 1368-1369 (2000).

19. Elias, D., Wolday, D., Akuffo, H., Petros, B., Bronner, U. \& Britton, S. Effect of deworming on human $\mathrm{T}$ cell responses to mycobacterial antigens in helminth-exposed individuals before and after bacille Calmette-Guérin (BCG) vaccination. Clin. Exp. Immunol. 123, 219-225 (2001).

20. Abate, E. et al. Effects of albendazole on the clinical outcome and immunological responses in helminth co-infected tuberculosis patients: a double blind randomised clinical trial. Int. J. Parasitol. 45, 133-140 (2015).

21. Chen, C.-C., Louie, S., McCormick, B., Walker, W. \& Shi, H. Helminthprimed dendritic cells alter the host response to enteric bacterial infection. J. Immunol. 176, 472-483 (2006).

22. Weng, M. et al. Alternatively activated macrophages in intestinal helminth infection: effects on concurrent bacterial colitis. J. Immunol. 179, 4721-4731 (2007)

23. Su, C.-W. et al. Duodenal helminth infection alters barrier function of the colonic epithelium via adaptive immune activation. Infect. Immun. 79, 2285-2294 (2011).

24. Li, R. et al. Alterations in the porcine colon microbiota induced by the gastrointestinal nematode Trichuris suis. Infect. Immun. 80, 2150-2157 (2012).

25. Walk, S., Blum, A., Ewing, S., Weinstock, J. \& Young, V. Alteration of the murine gut microbiota during infection with the parasitic helminth Heligmosomoides polygyrus. Inflamm. Bowel. Dis. 16, 1841-1849 (2010).
26. Su, C.-W. et al. Helminth infection impairs autophagy-mediated killing of bacterial enteropathogens by macrophages. J. Immunol. 189, 1459-1466 (2012).

27. Shi, H.N., Koski, K.G., Stevenson, M.M. \& Scott, M.E. Zinc deficiency and energy restriction modify immune responses in mice during both primary and challenge infection with Heligmosomoides polygyrus (Nematoda). Parasite Immunol 19, 363-373 (1997).

28. Chen, X. et al. A mouse model of Clostridium difficile-associated disease. Gastroenterology 135, 1984-1992 (2008).

29. Godinez, I. et al. Interleukin-23 orchestrates mucosal responses to Salmonella enterica serotype Typhimurium in the intestine. Infect. Immun. 77, 387-398 (2009).

30. Raffatellu, M. et al. Simian immunodeficiency virus-induced mucosal interleukin-17 deficiency promotes Salmonella dissemination from the gut. Nat. Med. 14, 421-428 (2008).

31. Zheng, Y. et al. Interleukin-22 mediates early host defense against attaching and effacing bacterial pathogens. Nat. Med. 14, 282-289 (2008).

32. Willing, B., Vacharaksa, A., Croxen, M., Thanachayanont, T. \& Finlay, B. Altering host resistance to infections through microbial transplantation. PLOS ONE 6, e26988 (2011).

33. Vallance, B.A., Deng, W., De Grado, M., Chan, C., Jacobson, K. \& Finlay, B.B. Modulation of inducible nitric oxide synthase expression by the attaching and effacing bacterial pathogen Citrobacter rodentium in infected mice. Infect. Immun. 70, 6424-6435 (2002).

34. Stecher, B. et al. Motility allows S. Typhimurium to benefit from the mucosal defence. Cell. Microbiol. 10, 1168-1180 (2008).

35. Ghosh, S. et al. Colonic microbiota alters host susceptibility to infectious colitis by modulating inflammation, redox status, and ion transporter gene expression. Am J Physiol Gastrointest Liver Physiol 301, G39-G49 (2011).

36. Knights, D., Lassen, K.G. \& Xavier, R.J. Advances in inflammatory bowel disease pathogenesis: linking host genetics and the microbiome. Gut 62 1505-1510 (2013).

37. Morgan, X.C. et al. Dysfunction of the intestinal microbiome in inflammatory bowel disease and treatment. Genome Biol. 13, R79 (2012).

38. Wang, Z. et al. Regulatory T cells promote a protective Th17-associated immune response to intestinal bacterial infection with C. rodentium. Mucosal Immunol. 7, 1290-1301 (2014).

39. Furusawa, Y., Obata, Y. \& Hase, K. Commensal microbiota regulates T cell fate decision in the gut. Semin. Immunopathol. 37, 17-25 (2015).

40. Reynolds, L.A. et al. Commensal-pathogen interactions in the intestinal tract: lactobacilli promote infection with, and are promoted by, helminth parasites. Gut Microbes 5, 522-532 (2014).

41. Zaiss, M.M. et al. The intestinal microbiota contributes to the ability of helminths to modulate allergic inflammation. Immunity 43, 1-13 (2015).

42. Rausch, S. et al. Small intestinal nematode infection of mice is associated with increased enterobacterial loads alongside the intestinal tract. PLOS ONE 8, e74026 (2013).

43. Maizels, R. et al. Immune modulation and modulators in Heligmosomoides polygyrus infection. Exp. Parasitol. 132, 76-89 (2012).

44. Grainger, J.R. et al. Helminth secretions induce de novo T cell Foxp3 expression and regulatory function through the TGF- $\beta$ pathway. J. Exp. Med. 207, 2331-2341 (2010).

45. Nagano, Y., Itoh, K. \& Honda, K. The induction of Treg cells by gutindigenous Clostridium. Curr. Opin. Immunol. 24, 392-397 (2012).

46. Lei, Y.M. et al. The composition of the microbiota modulates allograft rejection. J. Clin. Invest. 126, 2736-2744 (2016).

47. Shen, T.C. et al. Engineering the gut microbiota to treat hyperammonemia J. Clin. Invest. 125, 2841-2850 (2015).

48. Turnbaugh, P.J., Ridaura, V.K., Faith, J.J., Rey, F.E., Knight, R. \& Gordon, J.I. The effect of diet on the human gut microbiome: a metagenomic analysis in humanized gnotobiotic mice. Sci. Transl. Med. 1, 6ra14 (2009).

49. Bailey, M., Dowd, S., Parry, N., Galley, J., Schauer, D. \& Lyte, M. Stressor exposure disrupts commensal microbial populations in the intestines and leads to increased colonization by Citrobacter rodentium. Infect. Immun. 78, 1509-1519 (2010).

50. Dowd, S., Wolcott, R., Sun, Y., McKeehan, T., Smith, E. \& Rhoads, D. Polymicrobial nature of chronic diabetic foot ulcer biofilm infections determined using bacterial tag encoded FLX amplicon pyrosequencing (bTEFAP). PLOS ONE 3, e3326 (2008). 